\title{
Efeito da condição corporal ao parto sobre o desempenho produtivo de vacas mestiças Holandês $\times$ Zebu
}

\author{
José Esler de Freitas Júnior ${ }^{1}$, Vicente Ribeiro Rocha Júnior ${ }^{2}$, Francisco Palma Rennó ${ }^{3}$, \\ Marco Túlio Parrela de Mello ${ }^{1}$, Alexssandre Pinto de Carvalho ${ }^{1}$, Luciana Albuquerque Caldeira ${ }^{1}$ \\ ${ }^{1}$ Curso de Zootecnia do Departamento de Ciências Agrárias, Universidade Estadual de Montes Claros - UNIMONTES, R: Reinaldo Viana, \\ 2630, Bico da Pedra, Caixa Postal 91, CEP: 39440-000, Janaúba, MG. \\ 2 Departamento de Ciências Agrárias, Universidade Estadual de Montes Claros - UNIMONTES, R: Reinaldo Viana, 2630, Bico da Pedra, Caixa \\ Postal 91, CEP: 39440-000, Janaúba, MG. \\ ${ }^{3}$ Departamento de Nutrição e Produção Animal, Faculdade de Medicina Veterinária e Zootecnia da Universidade de São Paulo, Campus de \\ Pirassununga, SP.
}

RESUMO - Vinte e cinco vacas mestiças Holandês $\times$ Zebu foram utilizadas para avaliar o efeito da condição corporal ao parto sobre a produção e composição do leite e a mobilização de reservas corporais, avaliada por meio da variação da condição corporal do parto até os 120 dias de lactação. Os animais foram divididos em duas classes, de acordo com seu escore de condição corporal ao parto (ECCP), em uma escala de 1 (muito magra) a 5 (muito gorda): classe 1, vacas com ECCP $\geq 3,25$; e classe 2 , vacas com ECCP $<3,25$. As classes diferiram entre si quanto ao escore de condição corporal ao parto; as médias foram de 3,71 e 2,58, respectivamente, para as vacas das classes 1 e 2 . As vacas da classe 1 apresentaram maior mudança de escore de condição corporal (MECC) do parto aos 120 dias de lactação em relação às vacas da classe 2, com valores médios de $-0,57$ e - 0,12 unidades de escore de condição corporal para os grupos 1 e 2 , respectivamente. O escore de condição corporal ao parto não influenciou a produção de leite entre as classes 1 e 2 , no entanto, influenciou a produção de leite corrigida para 3,5\% de gordura, a porcentagem e a produção de gordura no leite. As vacas mestiças Holandês $\times$ Zebu apresentaram maior produção de gordura do leite e produção de leite corrigida para 3,5\% de gordura quando escore de condição corporal ao parto foi maior que 3,0 .

Palavras-chave: escore corporal, composição e produção do leite, mobilização de reservas corporais, vacas mestiças

\section{Effect of body condition score at calving on productive performance of crossbred Holstein-Zebu cows}

\begin{abstract}
Twenty-five crossbred Holstein-Zebu cows was used to evaluate the effect of body condition score at calving on milk yield and composition and evaluate the body reserves mobilization by variation of body condition score during the first 120 days of lactation. The cows were allocated in two classes according to the body condition score at calving (BCSC), using a scale from 1 (very thin) to 5 (very fat): class 1 , cows with of BCSC $\geq 3.25$; and class 2 , cows with BCSC $<3.25$. As expected the two classes of BCSC were significantly different at calving, showing mean values of 3.71 and 2.58 for class 1 and 2, respectively. From calving to 120 days of lactation cows of class 1 showed greater change in body condition score (CBCS) than cows of class 2 with mean cows, showed values of $-0,57$ and -, 12 units of BCS for class 1 and 2 , respectively. The body condition score at calving did not influence milk yield, but milk yield adjusted to $3.5 \%$ fat, fat percentage and production were different between cows of class 1 and 2. The crossbred cows Holstein-Zebu with a BCSC greater to 3.0 showed higher milk yield corrected to $3.5 \%$ fat and yield fat in this study.
\end{abstract}

Key Words: body reserve mobilization, body score, crossbred dairy cows, milk composition and yield

\section{Introdução}

A mobilização de tecidos corporais no período inicial de lactação consiste em uma fonte alternativa de energia de vacas leiteiras para suprir a demanda energética de mantença e a produção que não foi atendida pela dieta (Waltner et al., 1993). Segundo Tamminga et al. (1997), essa mobilização de reservas corporais, principalmente do tecido adiposo, pode suportar a produção de 120 a $550 \mathrm{~kg}$ de leite durante as primeiras semanas de lactação.

O monitoramento adequado das reservas corporais no período de transição é essencial para o manejo nutricional do rebanho, uma vez que vacas muito magras ou muito gordas ao parto estão predispostas a desordens metabólicas, 
problemas no parto, baixa produção e baixo desempenho reprodutivo, em virtude da mobilização excessiva de reservas corporais no início da lactação (Edmonson et al., 1989).

Apesar da natureza subjetiva, o método de avaliação de condição corporal por meio do escore de condição corporal (ECC) representa o meio mais barato, prático e não invasivo de quantificar as reservas depositadas ou mobilizadas do corpo do animal (NRC, 2001). Entre os diversos métodos de avaliação do escore de condição corporal em vacas leiteiras, destaca-se o método desenvolvido por Wildman et al. (1982) e Edmonson et al. (1989), baseado em avaliações visuais e táteis das reservas corporais em pontos específicos do corpo da vaca, em uma escala de 1 a 5 , com subunidades de 0,25 pontos.

Pedron et al. (1993) avaliaram o escore de condição corporal ao parto (ECCP) e as mudanças do escore de condição corporal (MECC) em relação à produção e composição do leite em vacas Holandesas e verificaram que vacas de maior escore de condição corporal ao parto mobilizaram maior quantidade de reservas corporais pós-parto. Esses autores afirmaram que estas reservas foram utilizadas para a produção de leite e não prejudicaram o desempenho reprodutivo.

Ângulo (1997) avaliou vacas mestiças e verificou maior MECC no início da lactação para vacas de maior escore de condição corporal ao parto. Segundo Ruegg \& Milton (1995), o padrão de mobilização de reservas corporais é influenciado pelo escore de condição corporal ao parto e pelo nível de produção, que altera a intensidade de mobilização de reservas corporais no início da lactação.

Domecq et al. (1997) verificaram que um ponto de aumento no ECC entre o período seco e o parto foi associado a acréscimo de $545,5 \mathrm{~kg}$ de leite nos primeiros 120 dias de lactação. Lago et al. (2001) avaliaram vacas da raça Holandesa e verificaram que os animais de maior escore de condição corporal ao parto $(\geq 4,0)$ perderam maior quantidade de reservas corporais no pós-parto, no entanto, não foi observada influência do escore de condição corporal ao parto sobre a incidência de doenças no período pós-parto. De forma semelhante, Gallo et al. (1996) verificaram que animais de maior condição corporal no início da lactação e de alta produção mobilizaram maiores quantidades de reservas corporais para produção.

Rennó et al. (2006) estudaram o efeito do ECC ao parto sobre a produção e composição do leite e constataram maior produção de leite e de seus componentes em vacas de ECC $\geq 3,25$ ao parto, resultados semelhantes aos obtidos por Pedron et al. (1993) e Waltner et al. (1993). Entretanto, Lago et al. (2001) não observaram efeito do ECC ao parto sobre a produção e o teor de gordura do leite durante os primeiros 120 dias de lactação.

A variação nos resultados encontrados na literatura sobre a influência do ECC ao parto sobre a produção de leite depende, segundo Lago et al. (2001), dos métodos adotados para avaliação da condição corporal e das diferenças de manejo e alimentação e do nível de produção dos rebanhos avaliados. Entretanto, considerando que no Brasil a maior parte do rebanho leiteiro é constituída de animais mestiços (Facó et al., 2002) e que existem poucos estudos sobre o balanço energético negativo e a mobilização de reservas corporais nesse tipo de animal (Borges et al., 2003), avaliou-se o efeito da condição corporal ao parto sobre a produção e o teor de gordura do leite, assim como a variação da condição corporal nos primeiros 120 dias de lactação em vacas mestiças Holandês $\times$ Zebu.

\section{Material e Métodos}

O experimento foi conduzido no Setor de Bovinocultura da Escola Agrotécnica Federal de Salinas, norte de Minas Gerais. Foram utilizadas 25 vacas mestiças Holandês $\times$ Zebu, que pariram entre fevereiro e outubro de 2005.

Os animais foram ordenhados mecanicamente duas vezes ao dia, às 8 e às $15 \mathrm{~h}$, e foram alimentados com concentrados em duas refeições diárias durante a ordenha. As dietas foram formuladas segundo recomendações do NRC (2001) utilizando-se silagem de sorgo como volumoso e concentrado à base de milho e farelo de soja.

Para avaliar o efeito da condição corporal ao parto sobre a produção e composição do leite e a variação da condição corporal no início da lactação, as vacas foram divididas em duas classes, de acordo com a condição corporal no dia do parto: classe 1, vacas com ECC $\geq 3,25$; e classe 2, vacas com ECC $<3,25$. Os escores de condição corporal ao parto foram de 3,71 e 2,58, com 12 e 13 repetições, para as classes 1 e 2 , respectivamente.

Durante o período experimental, quinzenalmente, do parto até 120 dias de lactação, coletaram-se amostras, procederam-se às pesagens da produção individual de leite e avaliaram-se as vacas quanto à condição corporal, realizada por um único avaliador utilizando-se a metodologia proposta por Wildman et al. (1982), desenvolvida por Edmonson et al. (1989), baseada em avaliações visuais e táteis das reservas corporais em pontos específicos do corpo do animal, utilizando-se uma escala biológica de 1 (muito magra) a 5 (muito gorda), com subunidades de 0,5 pontos. Efetuou-se ainda a coleta de amostra composta de leite, obtida a partir de duas amostras das duas ordenhas diárias, para análise do teor de gordura. 
Foram avaliados os escores de condição corporal ao parto (ECCP), a produção de leite (PL), a porcentagem de gordura $(\mathrm{G})$, a produção de gordura $(\mathrm{G} \mathrm{kg})$ e a mudança de escore de condição corporal (MECC) do parto aos 120 dias de lactação.

A produção de leite corrigida (PLC) para 3,5\% de gordura foi calculada utilizando-se a fórmula de Sklan et al. (1994):

PLCG 3,5\% = produção de leite $\times 0,432+0,163 \times \%$ gordura no leite

A análise do teor de gordura do leite foi realizada no laboratório da Escola Agrotécnica Federal de Salinas utilizando-se o método de separação por ácido sulfúrico. A produção de leite (PL), a produção de leite corrigida (PLC) para 3,5\% de gordura, a produção de gordura ( $\mathrm{G} \mathrm{kg}$ ), a porcentagem de gordura $(\mathrm{G} \%)$, o escore de condição corporal (ECC) e a mudança de escore de condição corporal (MECC) em relação ao escore de condição corporal ao parto foram analisadas pelo procedimento PROC MIXED do SAS (1999).

\section{Resultados e Discussão}

Durante o período experimental, a produção média de leite foi de $350 \mathrm{~L} /$ dia, considerando, em média, 25 vacas em lactação com produção de leite diária de $14,0 \mathrm{~kg} / \mathrm{vaca}$ (Tabela 2). A produção de leite não diferiu $(\mathrm{P}>0,05)$ entre as classes $1(E C C P \geq 3,25)$ e $2(E C C P<3,25)$ (Figura 1; Tabela 1), apesar de os animais de maior condição corporal ao parto terem apresentado tendência (Figura 1) de aumento da produção $(\mathrm{P}=0,06)$ nos primeiros 60 dias de lactação (Tabela 2).

Os animais de maior condição corporal ao parto apresentaram maior $(\mathrm{P}<0,10)$ produção de leite corrigida $(\mathrm{PLC})$ para $3,5 \%$ de gordura (Tabela 1; Figura 1), o que está de acordo com os resultados encontrados por Pedron et al. (1993) e Waltner et al. (1993), que verificaram maior PLC para as vacas de maior escore de condição corporal ao parto aos 120 e 90 dias de lactação, respectivamente, em animais de alta produção.

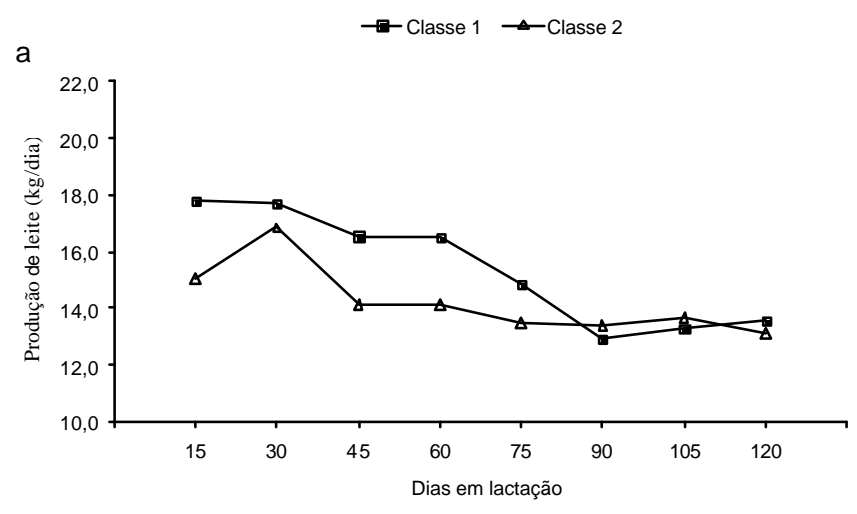

b

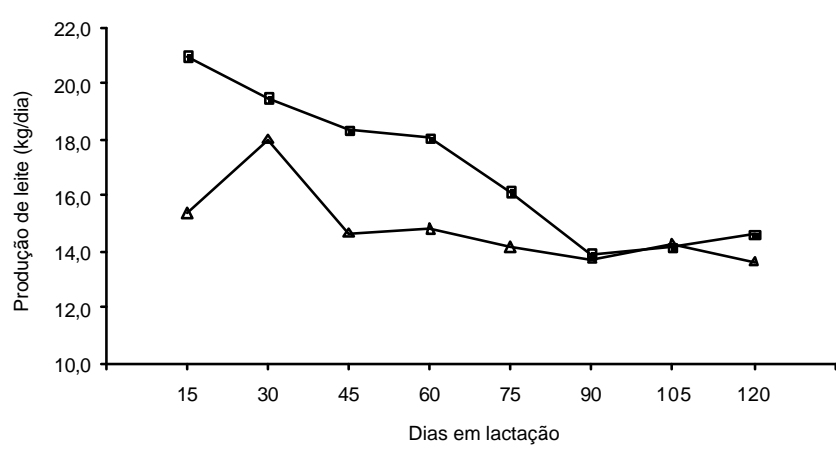

Figura 1 - Produção de leite (kg/dia) (a) e produção de leite corrigida para $3,5 \%$ de gordura $(\mathrm{kg} / \mathrm{dia})$ (b) durante a lactação de acordo com o escore de condição corporal ao parto $(\mathrm{ECCP}$ ) (classe $1 \mathrm{ECCP}=3,71$; classe $2 \mathrm{ECCP}=2,58$ )

Foram observadas diferenças na produção de gordura aos 15, 45 e 60 dias de lactação $(\mathrm{P}<0,05)$ e na porcentagem de gordura aos 15 dias pós-parto $(\mathrm{P}<0,05)$. Houve ainda tendência de diferença $(\mathrm{P}=0,074)$ aos 60 dias de lactação em comparação às classes de escore de condição corporal ao parto (Tabelas 1 e 2). As classes 1 e 2 apresentaram médias de 4,08 e 3,70\% de gordura no leite, respectivamente, do parto aos 120 dias de lactação (Figura 2), o que, segundo Pedron et al. (1993), pode ser atribuído à maior mobilização de gordura corporal na fase inicial da lactação, que proporciona nível elevado de ácidos graxos não esterificados na circulação, que podem ser utilizados pela glândula mamária

Tabela 1 - Produção de leite (PL), produção de leite corrigida para 3,5\% de gordura (PLC), produção (G kg) e porcentagem (G\%) de gordura, escore de condição corporal (ECC) e mudança de escore de condição corporal (MECC) com a classe de escore de condição corporal ao parto

\begin{tabular}{|c|c|c|c|c|c|c|}
\hline \multirow[t]{2}{*}{ Item } & \multicolumn{6}{|c|}{ Valor de P } \\
\hline & Produção de leite & PLC & Porcentagem de gordura $(\%)$ & Produção de gordura (kg) & $\mathrm{ECC}$ & Mudança de ECC \\
\hline Classe $^{1}$ & 0,216 & 0,073 & 0,054 & 0,048 & $<0,001$ & 0,005 \\
\hline Tempo ${ }^{2}$ & $<0,001$ & $<0,001$ & 0,778 & 0,004 & 0,127 & 0,126 \\
\hline Classe $\times$ tempo & 0,294 & 0,097 & 0,444 & 0,080 & 0,620 & 0,621 \\
\hline
\end{tabular}

${ }^{1}$ Classe 1: escore de condição corporal ao parto $\geq 3,25$ (média ECCP $=3,71$ ).

${ }_{1}^{1}$ Classe 2: escore de condição corporal ao parto $<3,25$ (média ECCP $=2,58$ ).

2 Dias em lactação. 
Tabela 2 - Produção de leite (PL), produção de leite corrigida para 3,5\% de gordura (PLC), produção (G kg) e porcentagem de gordura, escore de condição corporal (ECC) e mudança de escore de condição corporal (MECC) durante a lactação e entre as classes de escore de condição corporal ao parto

\begin{tabular}{|c|c|c|c|c|c|c|c|c|c|}
\hline \multirow[t]{2}{*}{ Item } & \multirow[t]{2}{*}{ Classe } & \multicolumn{8}{|c|}{ Dias de lactação } \\
\hline & & 15 & 30 & 45 & 60 & 75 & 90 & 105 & 120 \\
\hline \multirow{3}{*}{ Produção de leite (kg/dia) } & $1^{1}$ & 17,76 & 17,69 & 16,54 & 16,51 & 14,85 & 12,90 & 13,29 & 13,54 \\
\hline & $2^{2}$ & 15,05 & 16,85 & 14,15 & 14,15 & 13,48 & 13,39 & 13,69 & 13,11 \\
\hline & $\mathrm{P}<3$ & 0,039 & 0,496 & 0,093 & 0,060 & 0,196 & 0,674 & 0,737 & 0,750 \\
\hline \multirow{3}{*}{ Produção de leite corrigida (kg/dia) } & $1^{1}$ & 21,00 & 19,51 & 18,36 & 18,07 & 16,15 & 13,92 & 14,15 & 14,62 \\
\hline & $2^{2}$ & 15,40 & 18,03 & 14,68 & 14,85 & 14,21 & 13,75 & 14,30 & 13,66 \\
\hline & $\mathrm{P}<3$ & 0,007 & 0,391 & 0,035 & 0,044 & 0,139 & 0,909 & 0,936 & 0,568 \\
\hline \multirow{3}{*}{ Gordura (\%) } & $1^{1}$ & 4,52 & 4,11 & 4,16 & 4,07 & 4,03 & 3,94 & 3,82 & 4,01 \\
\hline & $2^{2}$ & 3,63 & 3,85 & 3,66 & 3,76 & 3,81 & 3,62 & 3,62 & 3,69 \\
\hline & $\mathrm{P}<3$ & 0,006 & 0,313 & 0,074 & 0,279 & 0,431 & 0,220 & 0,544 & 0,365 \\
\hline \multirow{3}{*}{ Gordura kg (kg/dia) } & $1^{1}$ & 0,818 & 0,729 & 0,690 & 0,672 & 0,599 & 0,512 & 0,515 & 0,537 \\
\hline & $2^{2}$ & 0,547 & 0,660 & 0,527 & 0,536 & 0,513 & 0,488 & 0,514 & 0,490 \\
\hline & $\mathrm{P}<3$ & 0,005 & 0,364 & 0,026 & 0,054 & 0,143 & 0,680 & 0,989 & 0,520 \\
\hline \multirow{3}{*}{ ECC } & $1^{1}$ & 3,29 & 3,25 & 3,12 & 3,12 & 3,17 & 3,21 & 3,17 & 3,29 \\
\hline & $2^{2}$ & 2,42 & 2,42 & 2,38 & 2,58 & 2,42 & 2,46 & 2,46 & 2,50 \\
\hline & $\mathrm{P}<3$ & 0,001 & $<0,001$ & $<0,001$ & 0,005 & $<0,001$ & 0,001 & 0,001 & $<0,001$ \\
\hline \multirow{3}{*}{ Mudança de ECC } & $1^{1}$ & $-0,42$ & $-0,46$ & $-0,58$ & $-0,58$ & $-0,54$ & $-0,50$ & $-0,54$ & $-0,42$ \\
\hline & $2^{2}$ & $-0,15$ & $-0,15$ & $-0,19$ & 0,00 & $-0,15$ & $-0,11$ & $-0,11$ & $-0,08$ \\
\hline & $\mathrm{P}<3$ & 0,111 & 0,044 & 0,009 & 0,002 & 0,012 & 0,032 & 0,022 & 0,024 \\
\hline
\end{tabular}

${ }_{1}$ Classe 1: escore de condição corporal ao parto $>=3,25$ (média ECCP=3,71).

${ }^{2}$ Classe 2: escore de condição corporal ao parto $<3,25$ (média $\mathrm{ECCP}=2,58$ ).

3 Nível de significância.

juntamente com os nutrientes provenientes da dieta para síntese de gordura do leite.

Desse modo, as vacas com maiores reservas de gordura corporal no pós-parto utilizaram em maior quantidade essas reservas para síntese de gordura do leite, em relação às vacas com menores depósitos de gordura corporal durante es ta fase da lactação.

A mudança no ECC diferiu significativamente $(\mathrm{P}<0,05)$ entre as classes 1 e 2 , indicando efeito do tempo sobre o ECC, afetado pelo escore de condição corporal ao parto (Tabelas 1 e 2). Os animais da classe 1 mobilizaram maior quantidade de reservas corporais do parto aos 120 dias de lactação (Figura 3 ) e apresentaram valores médios de - 0,57 a -0,12 unidades de ECC, respectivamente, para as classes 1 e 2 . O rebanho mestiço avaliado apresentou mobilização média de -0,35 unidades de ECC durante o período experimental. Outros autores (Ruegg \& Milton, 1995; Lago et al., 2001; Rennó et al., 2006) verificaram que os animais da raça Holandesa apresentaram mobilização de reservas corporais no pós-parto em torno de -0,80 unidades de ECC, indicando maior habilidade desses animais em mobilizar reservas corporais para produção de leite em comparação aos mes tiços Holandês $\times$ Zebu utilizados neste experimento.

Segundo Wildman et al. (1982), existe relação inversa entre o mérito genético da vaca e o valor de MECC, pois os animais de maior mérito genético mobilizam em maior quantidade as reservas corporais para produção. Os resultados obtidos neste trabalho com animais mestiços de média produção são semelhantes aos encontrados na literatura.

As vacas da classe 1, com média de ECC de 3,71, apresentaram ECC mínimo aos 50 dias de lactação (Figura 3), o que confirma os resultados encontrados por Domecq et al. (1997), cujo grupo de maior escore de condição corporal ao parto, com média de 2,77, apresentou o mínimo ECC aos 56 dias de lactação. Ruegg \& Milton (1995), ao avaliarem 429 vacas da raça Holandesa, verificaram que os animais de ECCP $\geq 4,00$ e com condição corporal entre 3,50 e 3,75 atingiram o menor ECC em torno dos 110 dias de lactação, em relação aos animais de $\mathrm{ECCP} \leq 3,25$, que apresentaram a menor condição corporal aos 50 dias de lactação.

De acordo com Gallo et al. (1996), a mudança no ECC após o parto ocorre de forma mais acentuada em animais de alta produção, em razão do maior grau de resistência dos tecidos de reserva à insulina no início da lactação, o que favorece a mobilização de reservas corporais para produção de leite. Ângulo (1997), avaliando vacas mestiças, verificou maior mudança no ECC de vacas de maior escore de condição corporal ao parto durante período maior da lactação. Pedron et al. (1993) observaram que os animais de ECCP>3,5 mobilizaram maior quantidade de reservas corpo- 


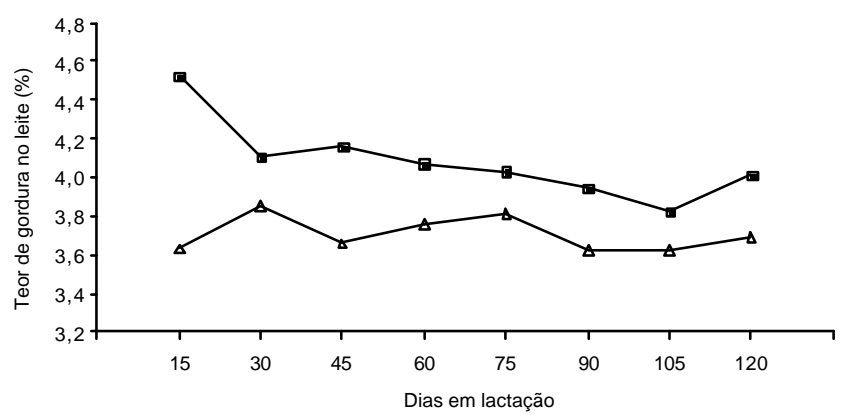

$\mathrm{b}$

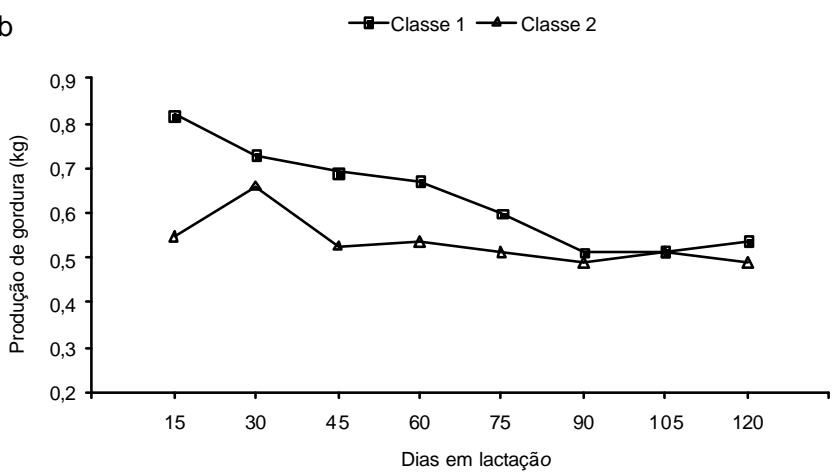

Figura 2 - Teor de gordura (a) e produção de gordura do leite (kg/dia) (b) durante a lactação, de acordo com o escore de condição corporal ao parto $(\mathrm{ECCP}$ ) (classe $1 \mathrm{ECCP}=3,71$; classe $2 \mathrm{ECCP}=2,58)$.

a

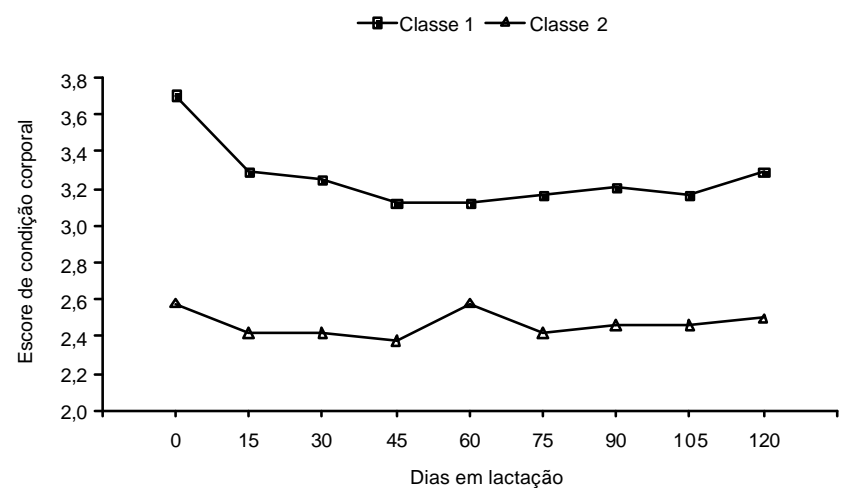

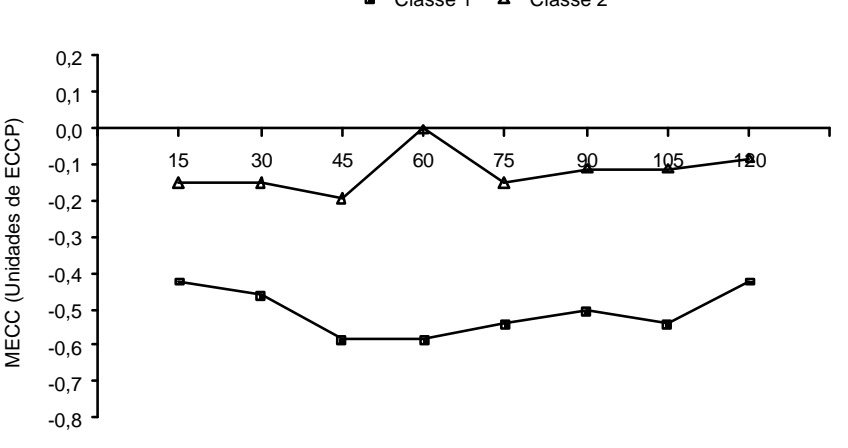

Dias em lactação

Figura 3 - Escore de condição corporal (a) e mudança de escore de condição corporal (MECC) (b) durante a lactação, de acordo com o escore de condição corporal ao parto (ECCP) (classe $1 \mathrm{ECCP}=3,71$; classe 2 ECCP $=2,58$ ).

rais após o parto e notaram ainda baixa taxa de insulina e aumento da taxa de lipólise.

\section{Conclusões}

O escore de condição corporal ao parto não influenciou a produção de leite, no entanto, os animais com maior escore mobilizaram maiores reservas corporais e produziram maior quantidade de gordura, o que resultou em maior produção de leite corrigida para $3,5 \%$ de gordura e em melhor condição corporal aos 120 dias de lactação.

\section{Literatura Citada}

ÂNGULO, L.A.M. Efeito da condição corporal ao parto a da produção de leite sobre o comportamento reprodutivo e variação de peso corporal no pós-parto de vacas mestiças leiteiras. Viçosa, MG: Universidade Federal de Viçosa, 1997. 57p. Dissertação (Mestrado em Zootecnia) - Universidade Federal de Viçosa, 1997.
BORGES, A.M.; RUAS, J.R.M.; ROCHA JR., V.R. Considerações sobre o manejo de fêmeas bovinas F1 e suas relações com as eficiências produtiva e reprodutiva. Informe Agropecuário v.25, n.221, p.47-55, 2004

DOMECQ, J.J.; SKIDMORE, A.L.; LLOYD, J.W. et al. Relationship between body condition scores and milk yield in a large herd of high yielding Holstein cows. Journal of Dairy Science, v.80, n.1, p.101-112, 1997.

EDMONSON, A.J.; LEAN, I.J.; WEAVER, L.D. et al. A body condition scoring chart for Holstein dairy cows. Journal of Dairy Science, v.72, n.1, p.68-78, 1989.

FACÓ, O.; LÔBO, R.N.B.; FILHO, R. et al. Análise do desempenho produtivo de diversos grupos genéticos Holandês-Gir no Brasil. Revista Brasileira de Zootecnia, v.31, n.5, p.1944-1952, 2002.

GALLO, L.; CARNIER, P.; CASSANDRO, M. et al. Change in body condition score of Holstein cows as affected by parity and mature equivalent milk yield. Journal of Dairy Science, v.79, n.6, p. 1009-1015, 1996.

LAGO, E.P.; PIRES, A.V.; SUSIN, I. et al. Efeito da condição corporal ao parto sobre alguns parâmetros do metabolismo energético, produção de leite e incidência de doenças no pós-parto de vacas leiteiras. Revista Brasileira de Zootecnia, v.30, n.5 p.15441549, 2001.

NATIONAL RESEARCH COUNCIL - NRC. Nutrient require ments of dairy cattle. 7.ed. Washington, D.C.: National Academic Press, 2001. 381p. 
PEDRON, O.; CHELI, F.; SENATORI, E. et al. Effect of body condition score on performance, some blood parameters, and milk fat, acid composition in dairy cows. Journalof Dairy Science, v.76, n.9, p.2528-2535, 1993.

RENNÓ, F.P.; PEREIRA, J.C.; SANTOS, A.D.F. et al. Efeito da condição corporal ao parto sobre a produção e composição do leite, curva de lactação e mobilização de reservas corporais em vacas da raça Holandesa. Arquivo Brasileiro de Medicina Veterinária e Zootecnia, v.58, n.2, p.220-233, 2006.

RUEGG, P.L.; MILTON, R.L. Body condition scores of Holstein cows on Prince Edward Island, Canadá: relationship with yield, reproductive performance and disease. Journal of Dairy Science, v.78, n.3, p.552-564, 1995.

SKLAN, D.; KAIM, M.; MOALLEM, U. et al. Effect of dietary calcium soaps on milk yield, body weight, reproductive hormones, and fertility in first parity and older cows. Journal of Dairy Science, v.70, n.11, p.3562-3577, 1992.

TAMMINGA, S.; LUTEIJN, P.A.; MEIJER, R.G.M. Changes in composition and energy content of live weight loss in dairy cows with time after parturition Livestock Production Science, v.52, p.31-38, 1997.

STATISTICAL ANALYSIS SYSTEM - SAS. User's guide: statistics. v. 8.0, Cary: 1999. (CD-ROM).

WALTNER, S.S.; McNAMARA, J.P.; HILLERS, J.K. Relationships of body condition score to production variables in high producing holstein dairy cattle. Journal of Dairy Science, v.76, n.11, p.3410-3419, 1993.

WILDMAN, O.E.E.; JONES, G.M. WAGNER, P.E. A dairy cow body condition scoring system and its relationship to selected production characteristics. Journal of Dairy Science, v.65, n.3, p.495-501, 1982 . 